\title{
Response of large-scale coastal basins to wind forcing: influence of topography
}

\author{
Wen L. Chen ${ }^{1}$ Pieter C. Roos ${ }^{1} \cdot$ Henk M. Schuttelaars ${ }^{2} \cdot$ Mohit Kumar $^{2}$. \\ Tjerk J. Zitman ${ }^{3}$ - Suzanne J. M. H. Hulscher ${ }^{1}$
}

Received: 30 June 2015 / Accepted: 23 January 2016 / Published online: 27 February 2016

(C) The Author(s) 2016. This article is published with open access at Springerlink.com

\begin{abstract}
Because wind is one of the main forcings in storm surge, we present an idealised process-based model to study the influence of topographic variations on the frequency response of large-scale coastal basins subject to time-periodic wind forcing. Coastal basins are represented by a semi-enclosed rectangular inner region forced by wind. It is connected to an outer region (represented as an infinitely long channel) without wind forcing, which allows waves to freely propagate outward. The model solves the three-dimensional linearised shallow water equations on the $f$ plane, forced by a spatially uniform wind field that has an arbitrary angle with respect to the along-basin direction. Turbulence is represented using a spatially uniform vertical eddy viscosity, combined with a partial slip condition at the bed. The surface elevation amplitudes, and hence
\end{abstract}

This article is part of the Topical Collection on Physics of Estuaries and Coastal Seas 2014 in Porto de Galinhas, PE, Brazil, 19-23 October 2014

Responsible Editor: Carlos Augusto França Schettini

Wen L. Chen

w.chen-3@utwente.nl

1 Water Engineering and Management, University of Twente, P.O. Box 217, 7500 AE Enschede, The Netherlands

2 Delft Institute of Applied Mathematics, Delft University of Technology, P.O. Box 5031, 2600 GA Delft, The Netherlands

3 Department of Hydraulic Engineering, Delft University of Technology, P.O. Box 5031, 2600 GA Delft, The Netherlands the vertical profiles of the velocity, are obtained using the finite element method (FEM), extended to account for the connection to the outer region. The results are then evaluated in terms of the elevation amplitude averaged over the basin's landward end, as a function of the wind forcing frequency. In general, the results point out that adding topographic elements in the inner region (such as a topographic step, a linearly sloping bed or a parabolic cross-basin profile), causes the resonance peaks to shift in the frequency domain, through their effect on local wave speed. The Coriolis effect causes the resonance peaks associated with cross-basin modes (which without rotation only appear in the response to cross-basin wind) to emerge also in the response to along-basin wind and vice versa.

Keywords Wind-driven flow · Coastal basins .

Resonance $\cdot$ Topography $\cdot$ Idealised process-based modelling $\cdot$ Coriolis effect $\cdot$ Frequency response

\section{Introduction}

Wind-driven set-up is the main contribution to extreme high water events, which may threaten coastal safety. This is particularly so when the combined characteristics of the wind forcing and the basin trigger resonance (Abraham 1960). A typical example is typhoon Winnie at the Korean coast of the Yellow Sea in 1997. The unusually strong and extensive coastal flooding was partly caused by resonant coupling of the Yellow Sea and the predominant period of the forcing (Moon et al. 2003).

Importantly, the resonance properties of coastal basins can be affected by large-scale topographic elements. For 
example, shoals may protect the coast (Hanley et al. 2013). Alternatively, the water body between a longshore bar and the coast may display strong oscillations, depending on the frequency of the incoming wave and local bathymetry (Büsching 2003). Moreover, wind blowing in different directions in a semi-enclosed basin may lead to significantly different responses (Breaker et al. 2010). For coastal safety, an overall practical goal is to be able to predict the wind-driven water levels at any location in basins of arbitrary shape and size. This requires physical insight in the influence of large-scale topography on resonance properties of large-scale coastal basins. Below, we will review the literature on this topic.

The influence of topography on surge response has been investigated in various site-specific studies using numerical models. For example, Chen et al. (2008) suggested that the record-high storm surge of Hurricane Katrina (New Orleans, Louisiana) was caused by the interaction of the surge with the extremely shallow, ancient deltaic lobe of the Mississippi river. For the surge caused by Hurricane Katrina, Irish et al. (2008) found that a milder shelf slope would have led to a higher surge. Alternatively, Weaver and Slinn (2010) found that small-scale variations in nearshore bathymetry of about $20 \%$ produce smaller variations in storm surge at the shoreline (less than $5 \%$ ). Using a one-dimensional numerical model, Libicki and Bedford (1990) showed how westward travelling storms over Lake Erie (approaching shallower regions) produce higher surge levels than eastward travelling storms (approaching deeper regions). Since these studies produce site-specific results, it is difficult to draw generic conclusions.

On the other hand, more generic studies focus on the frequency response of the systems to wind forcing. This is because a wind event can be seen as the superposition of periodic wind forcings at various frequencies $\omega$ (Craig 1989), i.e.

$\boldsymbol{\tau}_{w}(x, y, t)=\int_{-\infty}^{\infty} \hat{\boldsymbol{T}}_{\mathrm{w}}(x, y) \exp (-\mathrm{i} \omega t) \mathrm{d} \omega$,

where $\tau_{w}$ is wind stress, $\omega$ is the frequency and $\hat{\boldsymbol{T}}_{\mathrm{w}}(x, y)$ is the corresponding complex amplitude of wind forcing, which is in general a function of horizontal coordinates $x$ and $y$. Assuming linear dynamics, also the response, i.e. the flow and elevation pattern, will be the superposition of the responses at these individual frequencies. Hence, the basin's response to a wind event is contained in its so-called frequency response. Chen et al. (2015) applied this principle to different wind events over closed basins. They demonstrated that strong oscillations ('sloshing') occurs whenever the spectral response contains resonant peaks at frequencies that are excited by the wind event. Proudman (1929) provided analytical solutions for the response in narrow closed basins with a single topographic step. Alternatively, Ponte (2010) investigated the response of large-scale, elongated closed basins with a parabolic cross-basin topography to alongbasin wind forcing. Recently, Chen et al. (2015) extended this approach to closed basins with comparable length and width but restricted to uniform depth. Other studies focused on the eigenmodes of, e.g. closed basins with uniform depth (Rao 1966) or small-scale semi-enclosed basins with topography (Wilson 1972; Sobey 2005; Rabinovich 2009).

The goal of the present study is to investigate the influence of large-scale topography on the wind-driven frequency response of large-scale coastal basins, measured in terms of the set-up at the coast, and paying particular attention to the role of the Coriolis effect and wind angle. Here, large-scale means that we consider topographic elements with horizontal length scales of the scale of the basin, and that the basin is large enough for Coriolis effect to be important for phenomena with a time scale of the order of hours to days.

To achieve this goal, we have developed an idealised three-dimensional process-based model of a semi-enclosed rectangular rotating coastal basin subject to periodic wind forcing. The validity of linearization is debatable for very shallow regions. Therefore, we have made sure that the water depth is sufficiently large (10 m or more) in our examples. Furthermore, by considering topographic elements with horizontal length scales of the scale of the basin, we may safely ignore the associated nonlinearities (e.g. Csanady 1968; Mathieu et al. 2002; Winant 2004). The vertical profile of the flow field is resolved fully analytically and expressed in terms of the free surface elevation. In turn, the spatial pattern of free surface elevation amplitudes follows from solving an elliptic problem using the finite element method (FEM), extended to account for the connection of the coastal basin to the outer sea.

With this model, the frequency response of a coastal basin subject to spatially uniform periodic wind is investigated, both without and with the Coriolis effect. As a first step, we will consider a spatially uniform wind field with directions ranging from along-basin to cross-basin. This means that we ignore the dependency on $x$ and $y$ in Eq. 1 . The influence of topography on the frequency response is then investigated by systematically adding topographic details, expressing along-basin and cross-basin variations (to be detailed in Section 2.1 and Fig. 1). This is a first step which will be used in a follow-up study to investigate realistic storms.

This paper is organised as follows. In Section 2, we present the model. Next, Section 3 contains the solution method. The model results, showing the frequency responses for the various topographic elements introduced above, as well as the discussion are presented in Section 4. Finally, Section 5 contains the discussions and conclusions. 
(a) top view

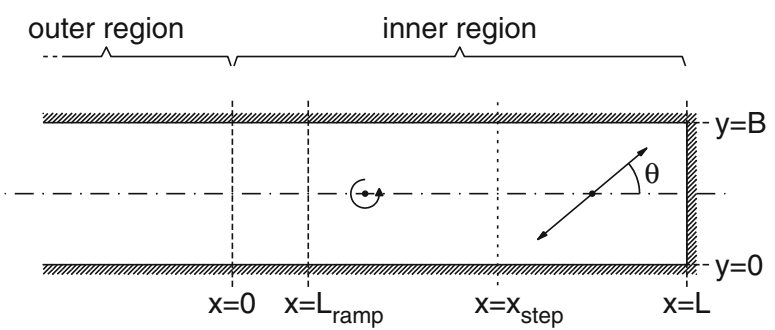

(b) side view

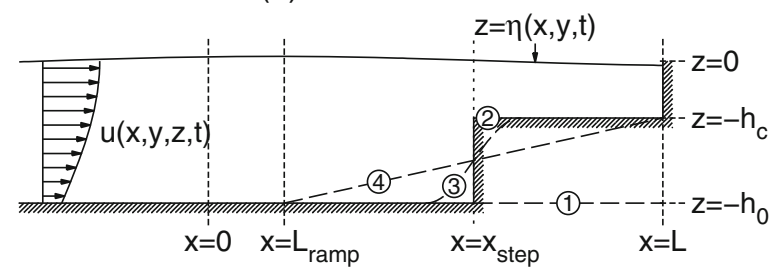

(c) ramp-up function

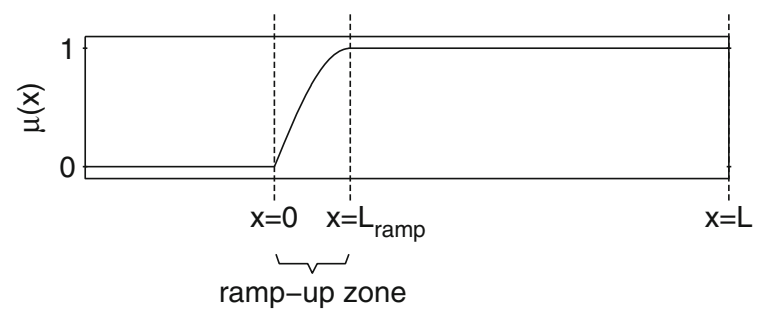

Fig. 1 Definition sketch of the model geometry of a rectangular rotating basin, subject to periodic wind forcing, which makes an angle $\theta$ with the along-basin direction. a Top view, showing the outer region, ramp-up region and inner region. b Side view in along-basin direction displaying the free surface elevation as well as vertical profile of the along-basin component of the three-dimensional flow field. The topography illustrated here is that of an abrupt step (type 2, here with $h_{\mathrm{c}}<h_{0}$ ), located at $x=x_{\text {step }}$. Dashed lines indicate three alternative topographies: uniform depth (type 1), smoothened step (type 3) and linear profile (type 4). For type 5, see Fig. 9. c Ramp-up function $\mu(x)$ used to describe the transition from no wind in the outer region to spatially uniform wind conditions in the core of the inner region

\section{Model formulation}

\subsection{Geometry}

Our model geometry consists of two parts: an inner region and an outer region (see Fig. 1).

- The inner region, of length $L$ and uniform width $B$, represents a rectangular semi-enclosed coastal basin. This is where the wind forcing takes place and where topographic elements will be added.

- The outer region, also of uniform width $B$, represents an outer sea. It stretches to infinity and experiences no wind forcing. By including the outer region, we allow wave energy to travel away from the inner region without reflecting at the interface with the outer region.

A ramp-up zone of length $L_{\mathrm{ramp}}$, part of the inner region, serves as a transition zone where the wind gradually increases from no wind in the outer region to a spatially uniform wind in the core of the inner region. This spatial transition is described by a so-called ramp-up function $\mu(x)$, to be introduced in Section 2.2 and to be further specified in Appendix A. Without ramp-up zone, a discontinuity would occur in the wind field, which would produce unrealistic model results.

The along-basin and cross-basin coordinates are denoted by $x$ and $y$, respectively, such that the closed boundaries are located at $x=L$ and $y=0, B$ and the interface between outer and inner region at $x=0$. The vertical coordinate $z$ points upward, with $z=\eta_{j}(x, y, t)$ denoting the free surface elevation with respect to the undisturbed water level $z=0$. The subscript $j=0$ represents the outer region, the inner region is labelled with $j=1$. The bottom topography is assumed to be spatially uniform in the outer region, and is denoted by $z=-h_{0}$. Over the inner region, the topography is allowed to vary, i.e. $z=-h_{1}(x, y)$. We will consider the following typical inner basin topographies (Fig. 1):

- uniform depth, which serves as a reference case (type 1 in Fig. 1). Note that Chen et al. (2015) also considered a uniform depth, but in a closed rather than a semi-enclosed basin.

- along-basin variations, such as a topographic step at $x=x_{\text {step }}$. Such a step divides the inner region into two subcompartments: an offshore part with the same uniform depth $h_{0}$ as the outer region, and a coastal part with depth $h_{\mathrm{c}}$. We will consider situations with a shallower coastal part $\left(h_{\mathrm{c}}<h_{0}\right.$, typical for many basins such as the Gulf of California and the Adriatic Sea) as well as with a deeper coastal part $\left(h_{\mathrm{c}}>h_{0}\right.$, as e.g. in the Norwegian trench). The topographic step can be both abrupt (type 2) and smoothened (type 3). In the latter case, the depth varies gradually from $h_{0}$ to $h_{\mathrm{c}}$ in a region of length $L_{\text {slope }}$ centred around $x=x_{\text {step }}$ (precise shape to be detailed in Section 4.4). Finally, we will also consider a linear profile from $h_{0}$ at $x=L_{\text {ramp }}$ to $h_{\mathrm{c}}$ at $x=L$ (type 4 ).

- cross-basin variations, such as a parabolic cross-basin profile with a smooth transition over a length $L_{\text {slope }}$ to the spatially uniform depth $h_{0}$ in the outer region and ramp-up zone (type 5). Most natural basins are deepest along their centerlines (as for the Gulf of California; see Ponte et al. 2012). This profile will be detailed in Section 4.6 and Fig. 9.

Our results will be quantified in terms of the water level amplitude, integrated along the coastline at the head of the 
basin (i.e., at $x=L$ ). This produces a scalar quantity, which reflects the basin's response along the entire basin head (instead of at a single point). This will be further specified in Section 4.1.

\subsection{Hydrodynamics}

Let $\boldsymbol{u}_{j}=\left(u_{j}, v_{j}, w_{j}\right)$ represent the flow velocity vector, with components $u_{j}, v_{j}$ and $w_{j}$ in the $x, y$ and $z$-direction, respectively $(j=0,1)$. Assuming that the vertical displacement of the free surface is small compared to the water depth, conservation of momentum and mass is expressed by the three-dimensional linearised shallow water equations on the $f$ plane according to

$$
\begin{aligned}
\frac{\partial u_{j}}{\partial t}-f v_{j} & =-g \frac{\partial \eta_{j}}{\partial x}+K \frac{\partial^{2} u_{j}}{\partial z^{2}}, \\
\frac{\partial v_{j}}{\partial t}+f u_{j} & =-g \frac{\partial \eta_{j}}{\partial y}+K \frac{\partial^{2} v_{j}}{\partial z^{2}}, \\
\frac{\partial u_{j}}{\partial x}+\frac{\partial v_{j}}{\partial y}+\frac{\partial w_{j}}{\partial z} & =0 .
\end{aligned}
$$

Here, $f=2 \Omega \sin \vartheta$ is the Coriolis parameter (with $\Omega=$ $7.292 \times 10^{-5} \mathrm{rad} \mathrm{s}^{-1}$ the angular frequency of the Earth's rotation and $\vartheta$ the latitude), $g=9.81 \mathrm{~m} \mathrm{~s}^{-2}$ the gravitational acceleration. Turbulence is represented using a spatially uniform and time-independent vertical eddy viscosity $K$ (e.g. Winant 2004; Ponte 2010), combined with a partial slip condition at the bed (e.g. Mass and Van Haren 1987; Hulscher 1996; Chernetsky et al. 2010). Horizontal mixing of momentum is neglected. The above linearisation further assumes that the effect of the advective terms around topographic elements can be neglected.

The kinematic and dynamic boundary conditions at the surface and bottom read, in linearised form:

$$
\begin{aligned}
& w_{j}=\frac{\partial \eta_{j}}{\partial t}, \quad K\left(\frac{\partial u_{j}}{\partial z}, \frac{\partial v_{j}}{\partial z}\right)=\frac{\left(\tau_{\mathrm{w}}^{(x)}, \tau_{\mathrm{w}}^{(y)}\right)}{\rho} \quad \text { at } z=0, \\
& w_{j}=0, \quad K\left(\frac{\partial u_{j}}{\partial z}, \frac{\partial v_{j}}{\partial z}\right)=s\left(u_{j}, v_{j}\right) \quad \text { at } z=-h_{j} .
\end{aligned}
$$

The linearisation procedure implies that the free surface condition in Eq. 5 is imposed at $z=0$ instead of at $z=\eta$. Furthermore, $\left(\tau_{\mathrm{w}}^{(x)}, \tau_{\mathrm{w}}^{(y)}\right)$ is the wind stress vector and $\rho$ the density of water. Assuming a frequency $\omega$ and a wind angle $\theta$ with respect to the along-basin direction, we write

$$
\frac{\left(\tau_{\mathrm{w}}^{(x)}, \tau_{\mathrm{w}}^{(y)}\right)}{\rho}=\mu(x) \hat{T}(\cos \theta, \sin \theta) \cos \omega t .
$$

Here, the constant $\hat{T}$ is the amplitude of the wind stress divided by the water density. The ramp-up function $\mu(x)$ introduced earlier, as sketched in Fig. 1, is specified in Appendix A.
In Eq. 6, we have introduced a constant resistance parameter $s$, its value usually obtained from the analysis of field data.

At the closed horizontal boundaries, we require zero normal transports, i.e.

$$
\left\langle u_{1}\right\rangle=0 \quad \text { at } x=L \quad \text { and } \quad\left\langle v_{j}\right\rangle=0 \quad \text { at } y=0, B,
$$

where $j=0,1$ and angle brackets denote vertical integration from bottom to surface, i.e. $\langle\cdot\rangle=\int_{-h_{j}}^{0} \cdot \mathrm{d} z$ (with the upper boundary $z=0$ arising from the linearisation).

At the interfaces between the adjacent regions, we require matching of surface elevation and normal transport:

$\eta_{0}=\eta_{1}, \quad\left\langle u_{0}\right\rangle=\left\langle u_{1}\right\rangle, \quad$ at $x=0$,

where we note that the water depth is continuous across both interfaces (and equal to $h_{0}$ ). Finally, regarding the outer region, we allow no wave energy coming in from infinity (Sommerfeld type of condition). This means that the solution in the outer region will be written as a superposition of outward propagating waves (in the negative $x$-direction). Because we include the Coriolis effect, these waves include Kelvin and Poincaré waves.

\section{Solution method}

\subsection{Preliminary considerations: wind angle}

The linearity of our model implies that the solution for arbitrary wind angle $\theta$ can be written as a linear combination of the solutions $\eta_{\text {along }}$ for along-basin wind $\left(\theta=0^{\circ}\right)$ and $\eta_{\text {cross }}$ for cross-basin wind $\left(\theta=90^{\circ}\right)$ :

$\eta(x, y, t)=\eta_{\text {along }}(x, y, t) \cos \theta+\eta_{\text {cross }}(x, y, t) \sin \theta$.

This similarly applies to the solution of the flow components $u, v$ and $w$. In our description of the solution method, we will therefore distinguish between along-basin and cross-basin wind only.

\subsection{Differential problem for surface elevation amplitude}

First, we write the solution in the outer and inner regions as time-periodic functions according to

$$
\begin{aligned}
\eta_{j}(x, y, t) & =\Re\left\{N_{j}(x, y) \exp (-\mathrm{i} \omega t)\right\}, \\
u_{j}(x, y, z, t) & =\Re\left\{U_{j}(x, y, z) \exp (-\mathrm{i} \omega t)\right\},
\end{aligned}
$$

with $\Re$ denoting the real part and with complex amplitudes $N_{j}$ and $U_{j}(j=0,1)$. Similar expressions hold for $v_{j}$ and $w_{j}$, with complex amplitudes $V_{j}$ and $W_{j}$.

Next, we express the horizontal flow solution $U_{j}$ and $V_{j}$ in terms of surface slopes $\nabla N_{j}$ and wind stress. Details of 
this derivation can be found in Appendix B. Substituting these expressions into the continuity equation and integrating from bottom to surface gives the following elliptic equation for $N$ :

$\nabla_{\mathrm{h}} \cdot\left[\left\langle\mathbf{D}_{j}\right\rangle \nabla_{\mathrm{h}} N_{j}\right]-\mathrm{i} \omega N_{j}=-\nabla_{\mathrm{h}} \cdot\left\langle\mathbf{r}_{j}\right\rangle$,

with horizontal nabla operator $\nabla_{\mathrm{h}}=(\partial / \partial x, \partial / \partial y)^{\mathrm{T}}$ as well as $2 \times 2$-matrix $\mathbf{D}_{j}$ and $2 \times 1$-vector $\mathbf{r}_{j}$, given by

$\mathbf{D}_{j}=\left[\begin{array}{cc}C_{j}^{+} & C_{j}^{-} \\ -C_{j}^{-} & C_{j}^{+}\end{array}\right], \quad \mathbf{r}_{j}=\left[\begin{array}{c}R_{j}^{+} \\ R_{j}^{-}\end{array}\right]$.

The coefficients $C_{j}^{+}$and $C_{j}^{-}$as well as the forcing terms $R_{j}^{+}$ and $R_{j}^{-}$depend on topography and thus on $x$ and $y$; they are specified in Appendix B.2.

The no normal transport conditions at the closed boundaries, as given by Eq. 8, imply

$$
\begin{gathered}
\left\langle C_{1}^{+}\right\rangle \frac{\partial N_{1}}{\partial x}+\left\langle C_{1}^{-}\right\rangle \frac{\partial N_{1}}{\partial y}=-\left\langle R_{1}^{+}\right\rangle \quad \text { at } x=L, \\
-\left\langle C_{j}^{-}\right\rangle \frac{\partial N_{j}}{\partial x}+\left\langle C_{j}^{+}\right\rangle \frac{\partial N_{j}}{\partial y}=-\left\langle R_{j}^{-}\right\rangle \quad \text { at } y=0, B .
\end{gathered}
$$

Finally, the vertical flow amplitude $W_{j}$ at any vertical position $z$ can be expressed in terms of the free surface elevation $N_{j}$ and wind forcing. This follows from vertical integration of the continuity equation (Appendix B.3).

The matching conditions at $x=0$, as expressed in Eq. 9, now becomes

$$
\begin{aligned}
N_{0} & =N_{1} \\
\left\langle C_{0}^{+}\right\rangle \frac{\partial N_{0}}{\partial x}+\left\langle C_{0}^{-}\right\rangle \frac{\partial N_{0}}{\partial y} & =\left\langle C_{1}^{+}\right\rangle \frac{\partial N_{1}}{\partial x}+\left\langle C_{1}^{-}\right\rangle \frac{\partial N_{1}}{\partial y} .
\end{aligned}
$$

Due to the continuity of the wind forcing across the interface, the $R_{j}^{+}$-contributions to the matching condition in Eq. 18 cancel.

\subsection{Finite element method}

The problem in Eqs. 13-16 for the elevation amplitude $N_{j}$ is solved by applying the finite element method (FEM, for the inner region), connected to a superposition of outward propagating waves (in the outer region).

To this end, the inner region is discretised into a set of $P$ triangular elements. The solution is then written as

$N_{1}(x, y)=\sum_{p=0}^{P} N_{1, p} \phi_{p}(x, y)$,

with coefficients $N_{1, p}$ and basis functions $\phi_{p}(x, y)$ which are either linear or quadratic polynomials with a value of 1 at node $\left(x_{p}, y_{p}\right)$ and 0 at all other nodes. To obtain the coefficients $N_{1, p}$, Eq. 13 is cast in weak form, and then integrated over the domain using test functions. Details can be found in Gockenbach (2006), and we also refer to Kumar et al. (2015), who developed a similar model to study tidal dynamics in estuaries.

The outer region deserves particular attention. Instead of applying a FEM-grid, the solution in the outer region is written as a truncated superposition of outward propagating waves. We thus write

$N_{0}(x, y)=\sum_{m=0}^{M} c_{0, m}^{\ominus} N_{m}^{\ominus}(y) \exp \left(\mathrm{i} k_{m}^{\ominus} x\right)$,

with coefficients $c_{0, m}^{\ominus}$. As indicated by the $\ominus$ superscript, this expression involves modes propagating in the negative $x$-direction only. Their cross-basin structures $N_{m}^{\ominus}(y)$, corresponding to a Kelvin mode $(m=0)$ and Poincaré modes $(m=1,2, \cdots)$, and the associated wave numbers $k_{m}^{\ominus}$ are specified in Appendix E. Because the individual modes satisfy the closed boundary conditions at $y=0, B$ in the outer region, so does the superposition in Eq. 20.

To satisfy the matching conditions at the interface at $x=$ 0 , we must connect the solutions (19) and (20) in the inner and outer region. This is done using a so-called collocation technique. We introduce a set of $M+1$ equidistant collocation points $(x, y)=\left(0, y_{m}\right)$ with $y_{m}=m B / M$ for $m=$ $0,1, \cdots, M$. Eqs. 17-18 are then applied at each of these collocation points, where the left-hand side follows from Eq. 20 and the right-hand side follows from interpolation of the FEM solution onto the collocation points.

This means that the FEM model is extended to account for waves radiating away from the inner region. Indeed, the linear matrix system contains conditions for the $P$ coefficients $N_{1, p}$ in Eq. 19 as well as conditions for the $M+1$ coefficients $c_{0, m}^{\ominus}$ in Eq. 20. Effectively, the solution in the outer region and the matching conditions at $x=0$ pose a non-reflecting boundary condition for the inner region, even in the presence of the Coriolis effect.

Finally, in special cases, the solution can be obtained by quick (semi-)analytical methods, which can furthermore be used to test the FEM model. We distinguish two cases:

- For $f=0$ and cross-basin wind $\left(\theta=90^{\circ}\right)$, the solution for uniform depth and the abrupt step topography can be found using a collocation technique also in the inner region $(\mathrm{COL})$. For the uniform depth case, the solutions in the ramp-up zone and the rest of the inner region are written as superpositions of a suitably chosen particular solution and two truncated families of $M+1$ wave modes (one Kelvin mode and $M$ Poincaré modes) propagating in the positive and negative $x$ direction, respectively. The particular solution is chosen to homogenise the boundary conditions at $y=0, B$ (which are nonhomogeneous due to the wind forcing). To satisfy the closed boundary condition at $x=L$ as well as the matching conditions between ramp-up zone and the rest of the inner region, we introduce two sets 
of $M+1$ collocation points at $x=L$ and $x=L_{\text {ramp, }}$, respectively. See Appendix C (and also Chen et al. 2015)

- A two-dimensional vertical (2DV) analytical solution can be found in the case without rotation $(f=0)$, with along-basin wind $\left(\theta=0^{\circ}\right)$, for the uniform depth or abrupt step topographies. This is detailed in Appendix D.

\section{Results and discussion}

\subsection{Introduction}

We consider a large-scale reference basin, with characteristics as shown in Table 1. To quantify the influence of wind and topography on the water levels along the coast in the basin, we define the amplification factor $A$ as the dimensionless elevation amplitude averaged over the right boundary at $x=L$, i.e.

$A=\frac{\overline{|N|}}{N_{\text {ref }}}$

with average amplitude $\overline{|N|}$ and reference amplitude $N_{\text {ref }}$ given by

$\overline{|N|}=\frac{1}{B} \int_{0}^{B}\left|N_{1}(L, y)\right| \mathrm{d} y, \quad N_{\mathrm{ref}}=\frac{\hat{T}}{g h_{\mathrm{c}} k_{\mathrm{c}}}$.

Here, $h_{\mathrm{c}}$ and $k_{\mathrm{c}}$ are the depth and wave number, respectively, that apply in the coastal part of the inner region (see Table 1 and Eq. 46 in Appendix D). For the uniform depth case, we take $h_{\mathrm{c}}=h_{0}$ and $k_{\mathrm{c}}=k_{0}$. Physically, the reference amplitude $N_{\text {ref }}$ follows from balancing the pressure gradient of a shallow water wave $g k_{\mathrm{c}} N_{\text {ref }}$ with the acceleration associated with the wind stress $\left(\hat{T} / h_{\mathrm{c}}\right)$.

In presenting the model results, the frequency response, expressed in the quantity $A$, is plotted as a function of a dimensionless forcing frequency $\omega / \omega_{\text {ref }}$, where the reference frequency is the frequency for which the shallow water wavelength of a basin with uniform depth equals the length of the basin:

$\omega_{\mathrm{ref}}=\sqrt{g h_{0}} \frac{2 \pi}{L}$

This remainder of this section is organised as follows. First, in Section 4.2, we show the influence of wind direction on the frequency response for the uniform depth. Then, in Section 4.3 and Section 4.4, we investigate the influence of step height for the abrupt step case as well as the influence of the slope length in the smoothened step case. Then, Section 4.5 and Section 4.6 contain the results for the linear along-basin slope and the parabolic cross-basin profile. An overview of test configurations is shown in Table 2.

\subsection{Uniform depth; influence of wind direction}

The influence of wind direction on frequency response is shown in Fig. 2. The colour plots show the amplification factor $A$ for non-rotating basins of uniform depth (topography type 1), as a function of the dimensionless frequency and wind angle. Note that wind angle is important as the open boundary introduces an essential difference between along-basin dynamics and cross-basin dynamics (contrasting the closed basin study by Chen et al. 2015). The response to along-basin wind has been obtained with the analytical 2DV solution, the response to cross-basin wind with the collocation solution (as outlined at the end of Section 3.3). Then, Eq. 10 has been applied to obtain the response to wind with an arbitrary angle.

The response to along-basin wind is independent of basin width. More specifically, the amplification factor $A$ is zero at $\omega / \omega_{\text {ref }} \approx 0,1, \cdots$ and local maxima in between.
Table 1 Overview of model parameters and their reference values

\begin{tabular}{llll}
\hline Description & Symbol & Value & Unit \\
\hline Basin width & $B$ & $100,(200,400)$ & $\mathrm{km}$ \\
Total basin length & $L$ & 400 & $\mathrm{~km}$ \\
Length of coastal part & $L_{\mathrm{c}}$ & 100 & $\mathrm{~km}$ \\
Water depth (outer region) & $h_{0}$ & 100 & $\mathrm{~m}$ \\
Water depth (coastal part) & $h_{\mathrm{c}}$ & $15^{\mathrm{a}}$ & $\mathrm{m}$ \\
Latitude & $\vartheta$ & $0^{\mathrm{b}}, 50^{\mathrm{c}}$ & ${ }^{\circ} \mathrm{N}$ \\
Vertical eddy viscosity & $K$ & 0.025 & $\mathrm{~m}^{2} \mathrm{~s}^{-1}$ \\
Resistance parameter & $s$ & $10^{-4}$ & $\mathrm{~m} \mathrm{~s}^{-1}$ \\
\hline
\end{tabular}

${ }^{a}$ Varied from $10-1000 \mathrm{~m}$ for the topographic step (topography type 2) and from $10-100 \mathrm{~m}$ for the linear profile (type 4)

${ }^{\mathrm{b}}$ Non-rotating basin

${ }^{\mathrm{c}}$ Strongly rotating basin 
Table 2 Overview of test configurations

\begin{tabular}{llll}
\hline Sec. & Topographic elements & Wind angle & Solution method \\
\hline Section 4.2 & Uniform depth (type $\left.1^{\mathrm{a}}\right)$ & $0^{\circ} \leq \theta \leq 90^{\circ}$ & 2DV, COL, FEM \\
Section 4.3 & Abrupt step $\left(2^{\mathrm{a}}\right)$ & $\theta=0^{\circ}$ & 2DV, FEM \\
Section 4.4 & Smoothened step (3 $\left.3^{\mathrm{a}}\right)$ & $\theta=0^{\circ}$ & 2DV, FEM \\
Section 4.5 & Linear profile $\left(4^{\mathrm{a}}\right)$ & $\theta=0^{\circ}$ & FEM \\
Section 4.6 & Parabolic profile $\left(5^{\mathrm{b}}\right)$ & $\theta=0^{\circ}$ & FEM
\end{tabular}

All test configurations include cases with and without Coriolis effect

${ }^{a}$ Along-basin topography types are shown in Fig. 1b

${ }^{\mathrm{b}}$ The cross-basin parabolic profile is to be presented in Fig. 9
This amplification pattern can be explained by systematically discussing the contributions to the solution in a onedimensional case (ignoring the ramp-up region by taking the limit $L_{\text {ramp }} \rightarrow 0$ and hence $\varphi_{\text {ramp }} \rightarrow 0$ in Appendix D).

The wind-driven flow at the closed boundary needs to be compensated by adding a contribution $\propto \cos (k[x-L])$ to the flow field, which together produce zero velocity at $x=L$ (and also zero elevation). If $k L=0,2 \pi, \cdots$, i.e. if $\omega / \omega_{\text {ref }}=p$, this superposition also has zero velocity and elevation at the open boundary $(x=0)$, by whichin this case-no outgoing wave occurs. On the other hand, if $k L \neq 0,2 \pi, \cdots$, this superposition has nonzero velocity and elevation at the open boundary $(x=0)$, which are 90 degrees out of phase and hence cannot be matched with an outgoing propagating wave at that interface. Hence, a second contribution $\propto \sin (k[x-L])$ must be added to the flow field in order to construct a solution at $x=0$ that can be matched by an outgoing propagating wave. Note that this leaves the velocity at $x=L$ unaffected. This second contribution to the velocity adds a nonzero contribution to the elevation at $x=L$, which is maximum for $k L=0,2 \pi, \cdots$, i.e. if $\omega / \omega_{\text {ref }}=p+\frac{1}{2}$, thus explaining the amplification pattern in the bottom panels of Fig. 2.

The slight deviation in the bottom panel of Fig. 2 from the exact integer values of $\omega / \omega_{\text {ref }}$ as explained above is due to the ramp-up of the wind forcing from $x=0$ to $x=L_{\text {ramp }}$. If the basin were closed at $x=0$ (instead of connected to an outer region where waves radiate away), the local maxima would be true resonance peaks with much higher values (Chen et al. 2015).

Since both boundaries in the lateral direction at $y=$ $0, B$ are closed, the response to cross-basin wind is much stronger than that to along-basin wind. Furthermore, the pattern strongly depends on basin width. To illustrate this, we choose three different width-to-length ratios, ranging from elongated $(B / L=1 / 4)$ to square $(B / L=1)$ (see
Fig. 2 Influence of wind angle on frequency response for nonrotating basin of uniform depth, for three width-to-length ratios: a $B / L=1 / 4, \mathbf{b} B / L=1 / 2$ and $\mathbf{c} B / L=1$. The colour plots show the amplification factor $A$ as a function of the dimensionless frequency $\omega / \omega_{\text {ref }}$ and wind angle $\theta$. The top and bottom panels show the frequency responses upon which the colour plots are based according to Eq. 10: $A_{\text {cross }}$ for cross-basin wind $\left(\theta=90^{\circ}\right.$, red $)$ and $A_{\text {along }}$ for along-basin wind $\left(\theta=0^{\circ}\right.$, blue $)$. As indicated by the different vertical scales, the peaks of the response to crossbasin wind are much higher than those for along-basin wind. Parameter values as in Table 1 (a) $B / L=1 / 4$
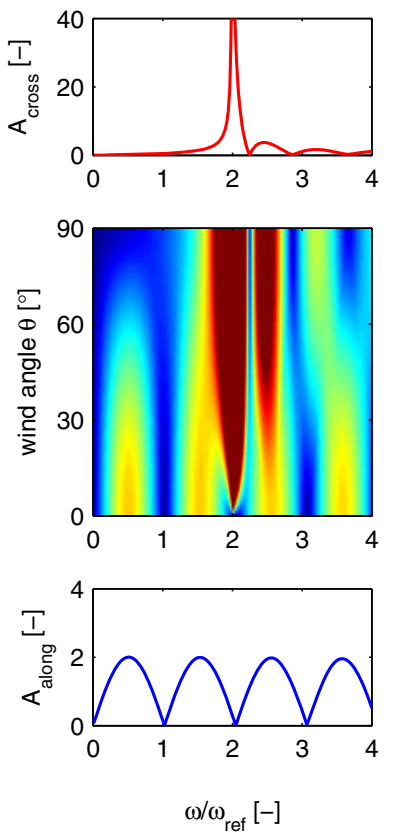

(b) $B / L=1 / 2$
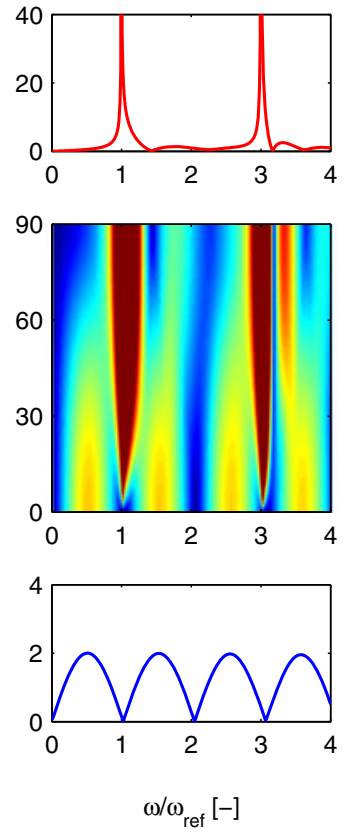

(c) $B / L=1$
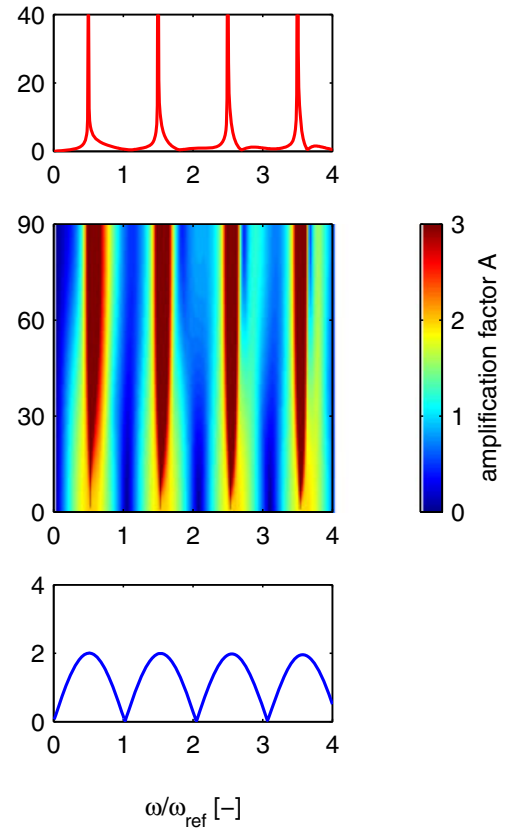
Fig. 2a-c). As a general result, the cross-basin response shows peaks at $\omega / \omega_{\text {ref }}=L / B(1 / 2,3 / 2,5 / 2 \cdots)$. These peaks are found to be cross-basin resonance (Chen et al. 2015). The cross-basin response is further influenced by the connection to the outer region, leading to small wiggles to the right of the peaks. This is different from a purely closed basin case (Chen et al. 2015). Finally, the gradual rampup of the wind field is crucial in the case of cross-basin wind. An abrupt transition at $x=0$ from no wind $(x<0)$ to full wind $(x>0)$ would produce unrealistically strong amplification around $(x, y)=(0,0)$ and $(0, B)$.

The colour plots in the middle panels of Fig. 2 show how the amplification factor $A$, according to Eq. 10, depends on the wind angle $\theta$. The response to cross-basin wind, due to its higher peaks, appears to dominate this pattern already for relatively small wind angles.

Figure 3 shows the influence of the Coriolis effect on the frequency response to cross-basin wind (top panels) and along-basin wind (bottom panels). Each plot contains a curve without rotation $\left(f=0\right.$ for $\left.\vartheta=0^{\circ}\right)$ and with rotation $\left(f \neq 0\right.$, as obtained for $\left.\vartheta=50^{\circ} \mathrm{N}\right)$. Because of the Coriolis-induced interaction between along-basin and cross-basin dynamics, the cross-basin peaks emerge also in the along-basin frequency responses. Further, peaks arise exactly in between the already existing peaks, e.g. at $\omega / \omega_{\text {ref }}=2$ in Fig. $3 b$, which corresponds to a cross-basin eigenmode. This mode, suppressed by symmetry for $f=0$, now emerges as the symmetry is broken by the Coriolis effect. Finally, both responses display a peak close to the inertial frequency $(f=\omega)$, which is invisible in the upper plots as the magnitude of the peaks is relatively small.

\subsection{Abrupt topographic step}

We will now investigate the influence of an abrupt topographic step, i.e. type 2 of the topographies introduced in Section 2.1 and Fig. 1. The solid lines in Fig. 4a show the frequency response for a non-rotating reference basin subject to along-basin wind. We present the following examples:

I Uniform depth (pink curve), with a depth of $h_{0}=$ $100 \mathrm{~m}$, which is in fact identical to the blue curve in the bottom panels of Fig. 2.

II Topographic step (black), i.e. the abrupt step case with a shallow coastal part of depth $h_{\mathrm{c}}=15 \mathrm{~m}$. Compared to the curve for uniform depth changes, we now see that the maxima become distorted and they furthermore shift to lower frequencies.

II' Same as example II, but now including the Coriolis effect (taking a latitude $\vartheta=50^{\circ} \mathrm{N}$ ). This case will be discussed further below.

The influence of step height is then investigated by varying the coastal depth $h_{\mathrm{c}}$ from 10 to $1000 \mathrm{~m}$, while keeping $h_{0}=100 \mathrm{~m}$. This leads to a depth ratio $h_{\mathrm{c}} / h_{0}$ ranging from 0.1 to 10 . The resulting frequency response for this range of $h_{\mathrm{c}} / h_{0}$ values is shown in Fig. $4 \mathrm{~b}$, where the red and blue colours indicate high and low amplification, respectively. The $h_{\mathrm{c}} / h_{0}$ values of examples I and II are indicated by the pink and black dashed horizontal lines, respectively.

The thick white lines follow local maxima of the amplification factor $|A|$, for increasing values of $h_{\mathrm{c}} / h_{0}$. In the bottom part of the figure, i.e. for $h_{c} / h_{0} \ll 1$, the peaks align with the thin solid white curves, for which the length $L_{\mathrm{c}}$ of the coastal part is an odd multiple of the quarter wave length on the coastal step. These lines are characterised by

$L_{\mathrm{c}}=\frac{1}{4}(2 p+1) \lambda_{\mathrm{c}}, \quad \lambda_{\mathrm{c}}=\frac{2 \pi}{k_{\mathrm{c}}}$,

for $p=0,1, \cdots$. Let us consider the case $p=1$, which corresponds to the three-quarter wavelength resonance of the (shallow) coastal part. For increasing $h_{\mathrm{c}}$ values, the leftmost thick white line at first follows this thin white line, but then shifts to a lower frequency. This is accompanied by an elevation node moving out of the coastal
Fig. 3 Influence of the Coriolis effect on frequency response to cross-basin wind (top panels) and along-basin wind (bottom panels), for three width-to-length ratios: a $B / L=1 / 4, \mathbf{b} B / L=1 / 2$ and $\mathbf{c}$ $B / L=1$. The thick background curves represent the responses of the non-rotating basin $\left(\vartheta=0^{\circ}\right)$, the sharp lines represent the rotating basin $\left(\vartheta=50^{\circ} \mathrm{N}\right)$. The vertical dashed line indicates the inertial frequency $\omega=f$. Parameter values as in Table 1 (a) $B / L=1 / 4$
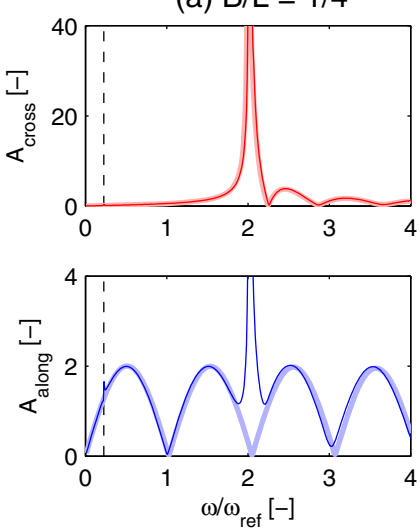

(b) $B / L=1 / 2$
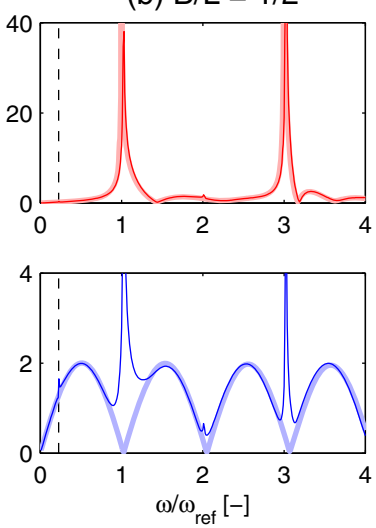

(c) $B / L=1$
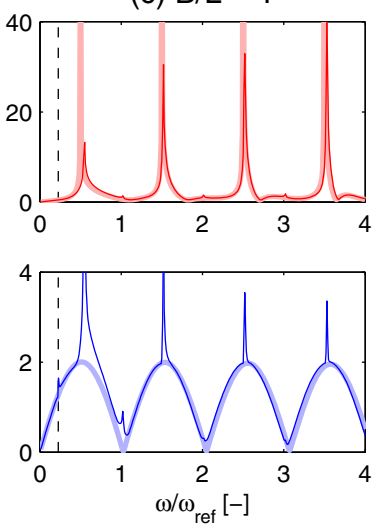
Fig. 4 Frequency response for a non-rotating basin with uniform depth and abrupt step. a Amplification factor $A$ as a function of dimensionless frequency $\omega / \omega_{\text {ref }}$ for two examples: (I) uniform depth (pink curve) and (II) topographic step (black). The dashed blue curve corresponds to example II' (same as example II but now including Coriolis effect). b Dependency of $A$ on depth ratio $h_{\mathrm{c}} / h_{0}$. Thick white lines follow the local maxima; four cases denoted by circle, square and triangles are further illustrated in Fig. 5. This figure has been obtained by varying $\omega$ and the depth of coastal part $h_{\mathrm{c}}$. Parameter values as in Table 1, with $\vartheta=0^{\circ}$ (a) examples of frequency response

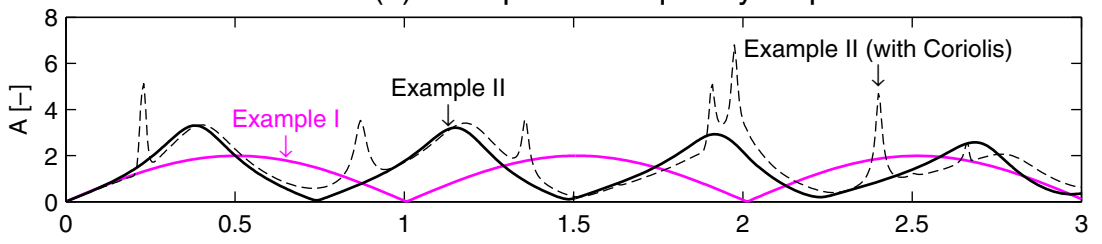

(b) influence of step height on A

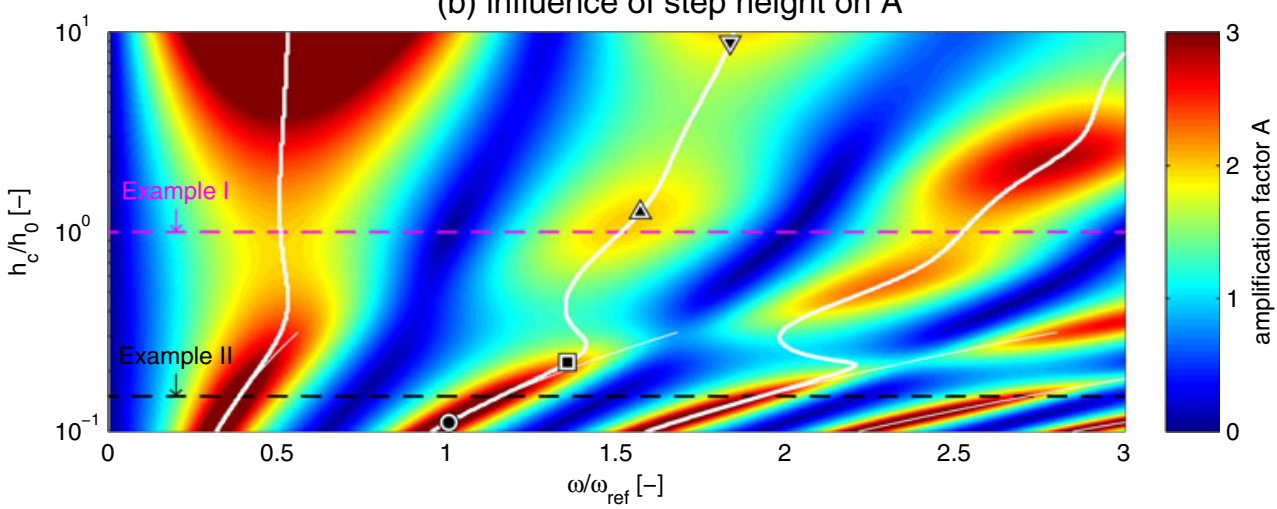

part because the wavelength in the coastal part becomes larger as $h_{\mathrm{c}}$ increases. This indicates the gradual transition towards a spatially uniform elevation pattern in the coastal part ('pumping mode'). This gradual transition is illustrated by the four plots in Fig. 5, showing the spatial structures of the elevation amplitudes for each of the four cases in Fig. 4. For the other thick white curves, obtained for $p=0$ and $p=2,3, \cdots$ in Eq. 24, this gradual process is similar but involves a different number of elevation nodes that subsequently move out of the coastal part. Note that, due to friction, the elevation nodes referred to here have a small nonzero amplitude rather than a zero value.

To illustrate the influence of the Coriolis effect on these results, Fig. 4 also contains the frequency response for example II', which is the same as example II but now in a rotating basin (latitude set to $\vartheta=50^{\circ} \mathrm{N}$ ). The Coriolis effect introduces new peaks, associated with cross-basin resonances in the (shallow) coastal part, with different along-basin and cross-basin structures. It should be noted that, to calculate this result in our FEM model, the abrupt step had to be smoothened. We chose a slope length of $L_{\text {slope }}=5 \mathrm{~km}$. The effect of smoothening topographic steps will be investigated in more detail in the next subsection.

\subsection{Influence of slope length (smoothened step)}

The topographic steps studied in Section 4.3 have a discontinuity in depth. In reality, however, such transitions are more gradual. To investigate this, we now consider a more smooth transition from $h_{0}$ to $h_{\mathrm{c}}$ over a length $L_{\text {slope }}$ and study this with the FEM model, restricting to a non-rotating basin $(f=0)$. The topography around the smoothened step, as depicted in Fig. 1 (type 3), is written as

$h_{1}(x)=h_{0}+\left(h_{\mathrm{c}}-h_{0}\right) F\left(x-x_{1}\right)$,
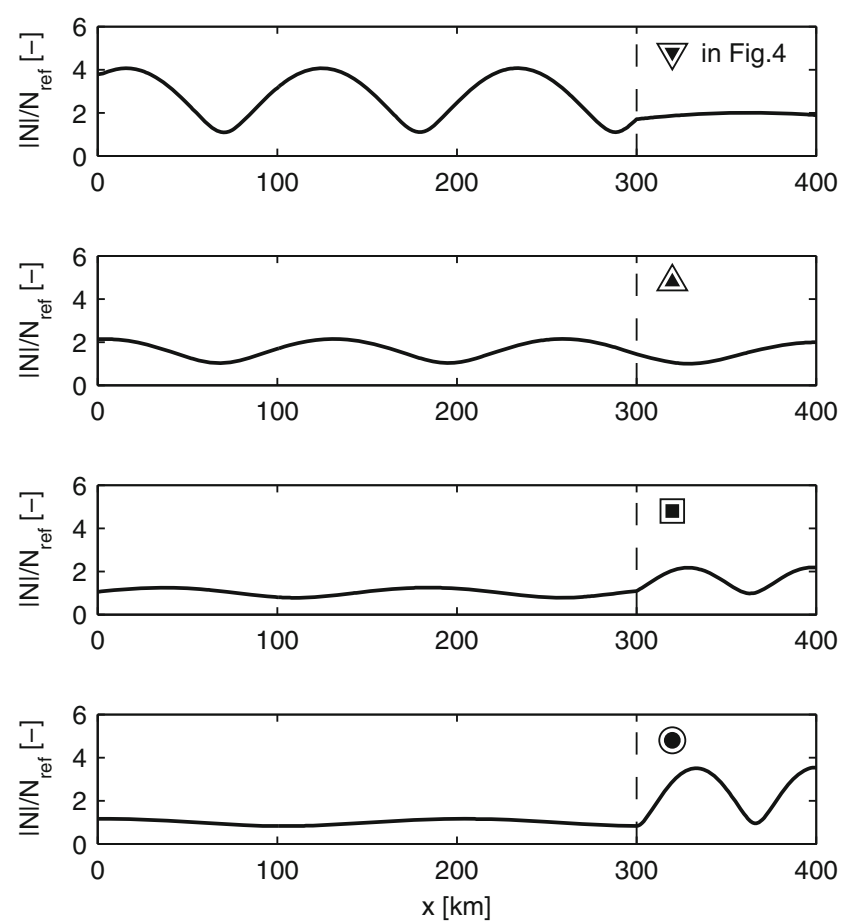

Fig. 5 Spatial structure of the absolute value of the dimensionless elevation amplitude $|N(x)| / N_{\text {ref }}$ for the four cases denoted by a circle, a square, an upward pointing triangle and a downward triangle in Fig. 4. The vertical dashed line indicates the position of the topographic step. For further explanation, see text. The ramp-up region has a length of $L_{\text {ramp }}=10 \mathrm{~km}$ 
Fig. 6 Influence of slope length on frequency response. a Amplification factor $A$ as a function of dimensionless frequency $\omega / \omega_{\text {ref }}$ and slope length $L_{\text {slope }}$, as obtained with our FEM model. b Frequency response for an abrupt topographic step $\left(L_{\text {slope }}=0\right)$, as obtained with the 2DV solution method, as indicated by the black dashed curve in Fig. 4. Parameter values: $h_{\mathrm{c}}=15 \mathrm{~m}$, $h_{0}=100 \mathrm{~m}$

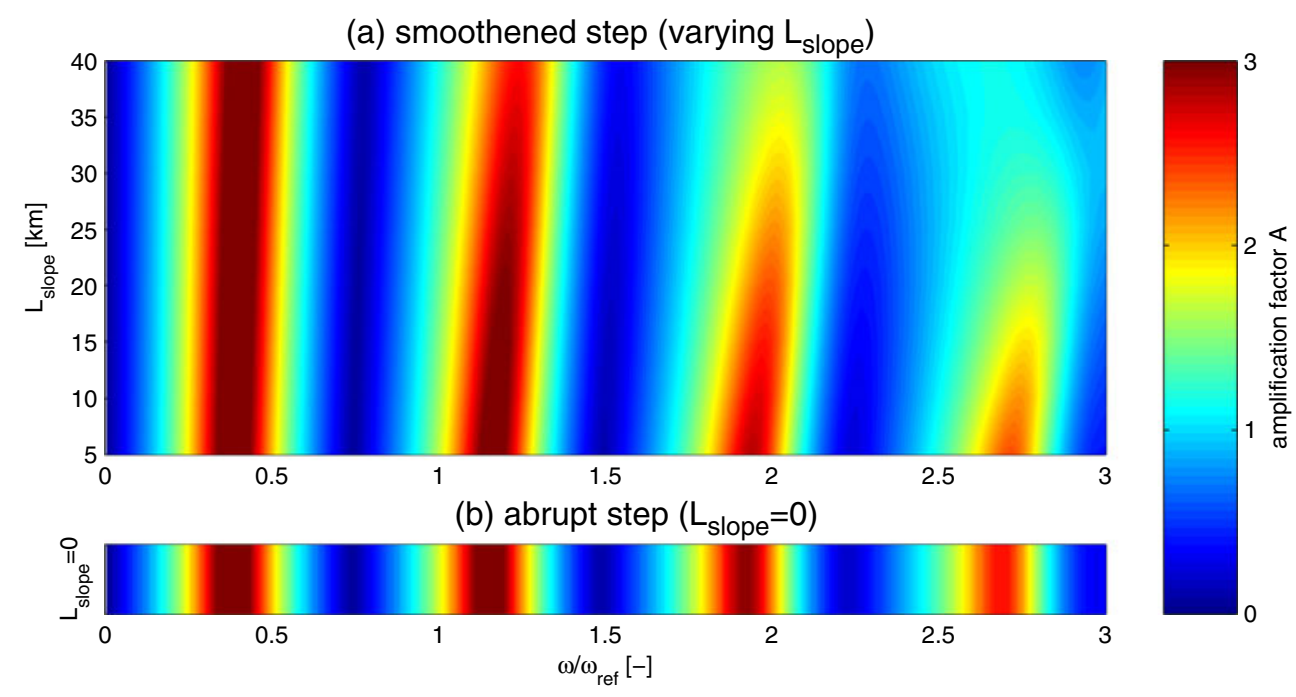

with dimensionless transition function $F(\xi)$ chosen to be of sinusoidal shape:

$F(\xi)=\frac{1}{2}+\frac{1}{2} \sin \left(\frac{\pi \xi}{L_{\text {slope }}}\right), \quad-\frac{L_{\text {slope }}}{2} \leq \xi \leq \frac{L_{\text {slope }}}{2}$.

Figure 6a shows the influence of varying the slope length $L_{\text {slope }}$ on the frequency response, as obtained with our FEM model. We again consider the reference depths $h_{0}=100 \mathrm{~m}$ and $h_{\mathrm{c}}=15 \mathrm{~m}$ as given in Table 1 and $L_{\text {slope values rang- }}$ ing from 5 to $40 \mathrm{~km}$. Importantly, the average depth of the inner region is the same for all $L_{\text {slope values. The result }}$ shows that, for increasing values of $L_{\text {slope }}$, the frequency of the peaks shift to slightly higher values. This shift can be explained as follows. The more smooth the step (while maintaining the average depth), the larger the basin average of $\sqrt{g h}$ will be, which is a proxy for the wave speed in the basin. Such an increase in effective wave speed reduces the travel time of waves around the basin, which implies that resonance occurs at higher frequencies.
Finally, in the limit of very small slope lengths, the frequency response as obtained with our FEM model converges to the results of the abrupt topographic step studied in Section 4.3 (see Fig. 6b). On the other hand, for very large slope lengths, the step becomes so gradual that it resembles a linear profile, to be studied next.

\subsection{Linear profile}

The influence of a bed with a linear along-basin slope in the inner region (type 4) on the frequency response of a coastal basin, restricting to along-basin wind, is investigated by fixing the depth in the outer and ramp-up region to its reference value $h_{0}=100 \mathrm{~m}$. The depth $h_{\mathrm{c}}$, that is attained at the coast $(x=L)$, is then varied from 100 to $10 \mathrm{~m}$. Maintaining uniformity in the cross-basin direction, the bed slope $S$ in the inner region is thus given by

$S=\frac{h_{0}-h_{\mathrm{c}}}{L-L_{\mathrm{ramp}}}$
Fig. 7 Influence of the bed slope in the inner region on the frequency response to along-basin wind. Amplification as a function of dimensionless frequency $\omega / \omega_{\text {ref }}$ and bed slope $S=\left(h_{0}-h_{\mathrm{c}}\right) /\left(L-L_{\mathrm{ramp}}\right)$ in two cases: a without the Coriolis effect and $\mathbf{b}$ with the Coriolis effect. For $S=1.75 \times 10^{-4}$, the spatial elevation patterns of three cases, i.e., $\omega / \omega_{\text {ref }}=1.8$ (indicated by a triangle in Fig. 7b), $\omega / \omega_{\text {ref }}=2.1$ (square) and $\omega / \omega_{\text {ref }}=2.31$ (circle), are plotted in Fig. 8 (a) without Coriolis effect $\left(\vartheta=0^{\circ}\right)$

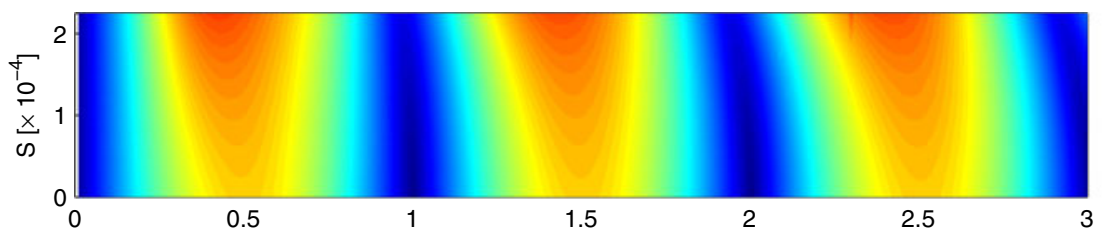

(b) with Coriolis effect $\left(\vartheta=50^{\circ} \mathrm{N}\right)$

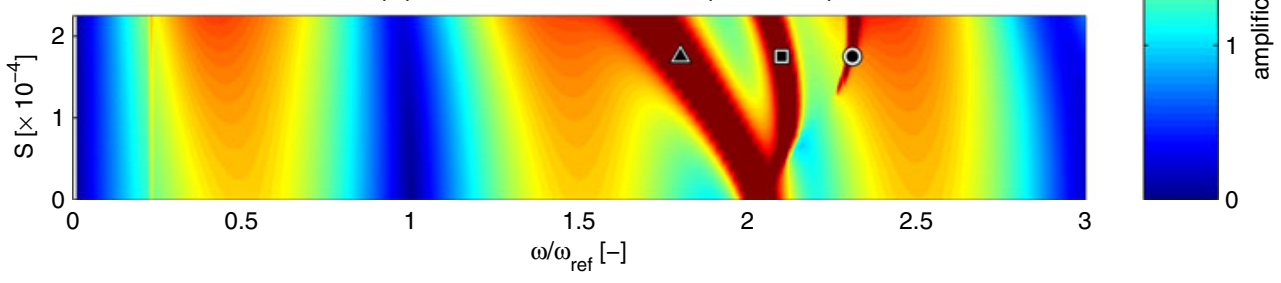


Fig. 8 Spatial structure of the absolute value of the dimensionless elevation amplitude $|N(x, y)| / N_{\text {ref }}$ for the three cases, i.e., a $S=1.75 \times 10^{-4}, \omega / \omega_{\text {ref }}=1.8$ (triangle in Fig. 7b), b $S=1.75 \times 10^{-4}, \omega / \omega_{\text {ref }}=2.1$ (square in Fig. 7b) and c $S=1.75 \times 10^{-4}, \omega / \omega_{\text {ref }}=2.3$ (circle in Fig. 7b) (a) $S=1.75 \times 10^{-4}, \omega / \omega_{\text {ref }}=1.8$ (triangle in Fig. $7 b$ )

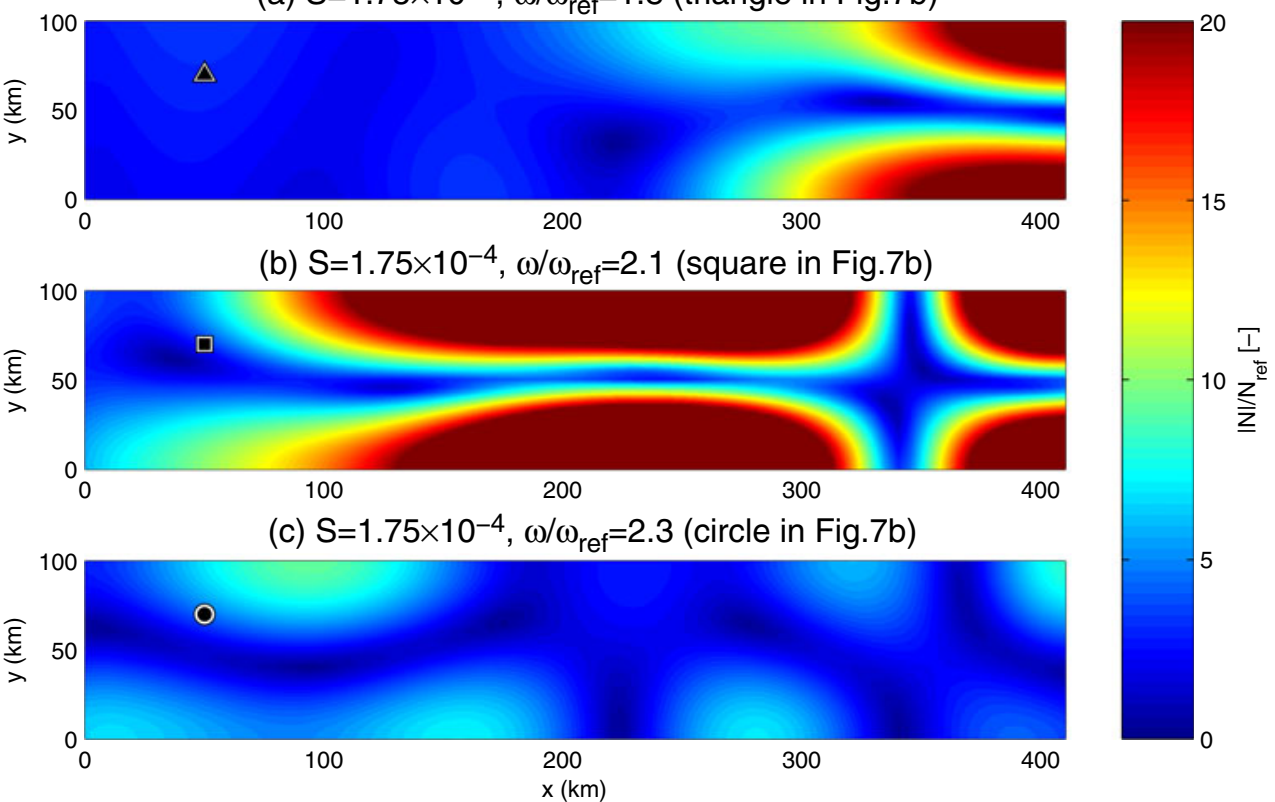

Figure 7 shows the frequency responses, both with and without Coriolis effect, obtained with the FEM model. The responses for zero slope $(S=0)$ are identical to the curves in the bottom plot of Fig. 3a. With the Coriolis effect, the frequency response includes peaks associated with crossbasin resonances, the frequency of which becomes smaller as $S$ increases. Furthermore, as the slope is increased, more peaks appear that are also associated with cross-basin resonances, but differ in their along-basin structures (see Fig. 8). Away from these resonance peaks and regardless of the Coriolis effect, increasing the bed slope leads to higher amplification, the maxima of which are shifted to slightly lower frequencies. For increasing slope parameter $S$, the peaks move to lower frequencies because the average depth decreases, which effectively slows down the wave propagation. Then, the splitting into more branches is due to the fact that for increasing $S$, the basin covers a wider range of depth values. As a result, cross-basin resonances may occur at different locations along the basin axis.

\subsection{Parabolic cross-basin profile}

Finally, we investigate the influence of a parabolic crossbasin depth profile (type 5) on the frequency response, restricting to along-basin wind. The shape, depicted in Fig. 9, is given by

$h(x, y)=h_{0}+\Delta h F\left(x-x_{2}\right)\left[1-12\left(\frac{y}{B}-\frac{1}{2}\right)^{2}\right]$,

with parameter $\Delta h$, dimensionless transition function $F(\xi)$ as already introduced in Eq. 26, to be centred around $x=x_{2}$ with $x_{2}=L_{\text {ramp }}+\frac{1}{2} L_{\text {slope }}$. The profile is chosen such that the width-averaged depth is equal to $h_{0}$. The depth at the centerline is given by $h_{0}+\Delta h$, the depth at the banks $y=$ $0, B$ is given by $h_{0}-2 \Delta h$. Importantly, the dimensionless transition function $F(\xi)$ ensures a smooth transition from the spatially uniform depth $h_{0}$ in the outer region and rampup region to our parabolic cross-basin profile in the inner region.

(a) along-basin side view

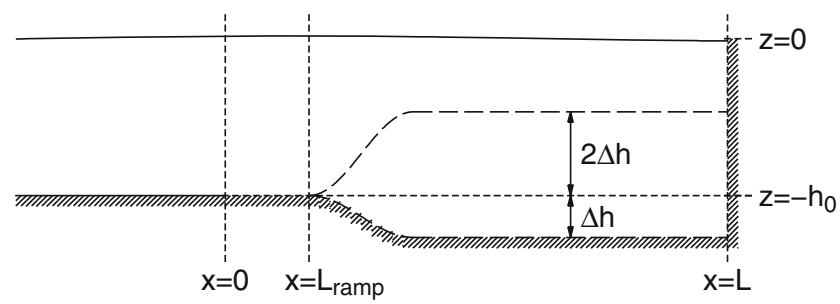

(b) cross-basin side view

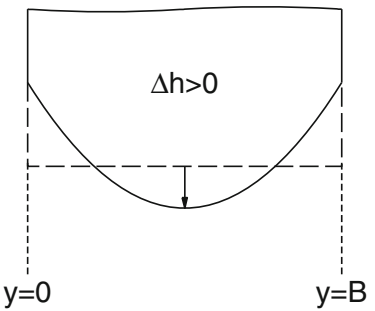

Fig. 9 Definition sketch of parabolic profile. a Along-basin side view showing gradual transition from uniform depth $h_{0}$ in outer and rampup region to parabolic cross-basin profile in inner region. The dashed lines at $z=-h_{0}-\Delta h$ and $z=-h_{0}+2 \Delta h$ indicate the water depth at the centerline and the banks, respectively. b Cross-basin side view in the inner region, showing the parabolic profile according to Eq. 28 
Using the reference value $h_{0}=100 \mathrm{~m}$, we vary $\Delta h$ from 0 to $40 \mathrm{~m}$, giving a ratio $\Delta h / h_{0}$ between 0 and 0.4 . Figure 10 shows the frequency responses, both with and without Coriolis effect, obtained with the FEM model. The responses for $\Delta h=0$, corresponding to a uniform depth, are in fact identical to the curves in the bottom plot of Fig. 3a.

Without Coriolis effect, for increasing values of $|\Delta h|$, the local maxima of the amplification shift to lower frequencies. This can be explained by the effective wave speed that is based on the cross-basin average of $\sqrt{g h}$, as mentioned in Section 4.4. Indeed, the result of increasing $|\Delta h|$ is qualitatively similar to that of reducing the slope length $L_{\text {slope }}$ for the smoothened step case. The results with the Coriolis effect show a similar 'background' amplification pattern as obtained for $\vartheta=0^{\circ}$. In addition to that, the Coriolis effect introduces peaks associated with cross-basin resonances with peak frequencies that shift to higher values for increasing $\Delta h$. The circulating wave over a parabolic profile is more confined to the centerline than over a flat bottom, which effectively reduces the travelling distance leading to a higher resonant frequency.

\section{Discussions and conclusions}

We have developed an idealised process-based model to analyse the influence of specific topographic elements on the frequency response of semi-enclosed coastal basins subject to time-periodic wind forcing. Coastal basins are represented by a large-scale semi-enclosed rectangular inner region where the wind forcing takes place and where a variety of topographic elements have been included. It is connected to an outer region, without wind forcing and stretching to infinity, which allows waves to freely propagate outward. The model solves the three-dimensional linearised shallow water equations on the $f$ plane, forced by a wind field that ramps up to a spatially uniform pattern in the core of the inner region. The wind field has an arbitrary angle with respect to the along-basin direction. The model solves a two-dimensional problem for the surface elevation amplitudes by applying the finite element method (FEM), extended to account for the outward propagating waves in the outer region. In particular cases, alternative (semi)analytical solution techniques are used. Strictly speaking, a FEM approach allowing for unstructured grids is not necessary for the geometries considered in this study. However, this FEM model allows us to consider more complicated geometry and topography in later studies.

By restricting to linear dynamics, we have neglected nonlinear effects. This facilitates our understanding in two ways: $(i)$ the response to any wind event is contained in the frequency response (as will be discussed further below) and (ii) the frequency response for arbitrary wind angle is in fact a linear combination of the frequency responses to alongbasin and cross-basin wind. The responses to along-basin and cross-basin wind are essentially different because of the differences in the along-basin and cross-basin dynamics caused by the open boundary.

To analyse our model, we focused on the elevation amplitude averaged over the basin's landward end, as a function of the dimensionless frequency $\omega / \omega_{\text {ref }}$ with reference frequency $\omega_{\text {ref }}=\sqrt{g h_{0}} 2 \pi / L$ for which the shallow water wavelength (of the basin with uniform depth) equals the length of the basin. For cases not including the Coriolis effect, we conclude the following.

1. In the reference case of a uniform depth, the response to along-basin wind is a pattern showing zero amplification at $\omega / \omega_{\text {ref }} \approx 0,1,2, \cdots$ and maximum amplification in between. Due to the waves allowed to propagate away into the outer sea, these maxima are found to be much weaker than the peaks in the frequency response to cross-basin wind.

2. For a topographic step with a shallow coastal part, we observe the resonance frequencies associated with (odd
Fig. 10 a, b Same as Fig. 7, but now the influence of parabolic cross-basin profile on the frequency response as a function of $\Delta h / h_{0}$

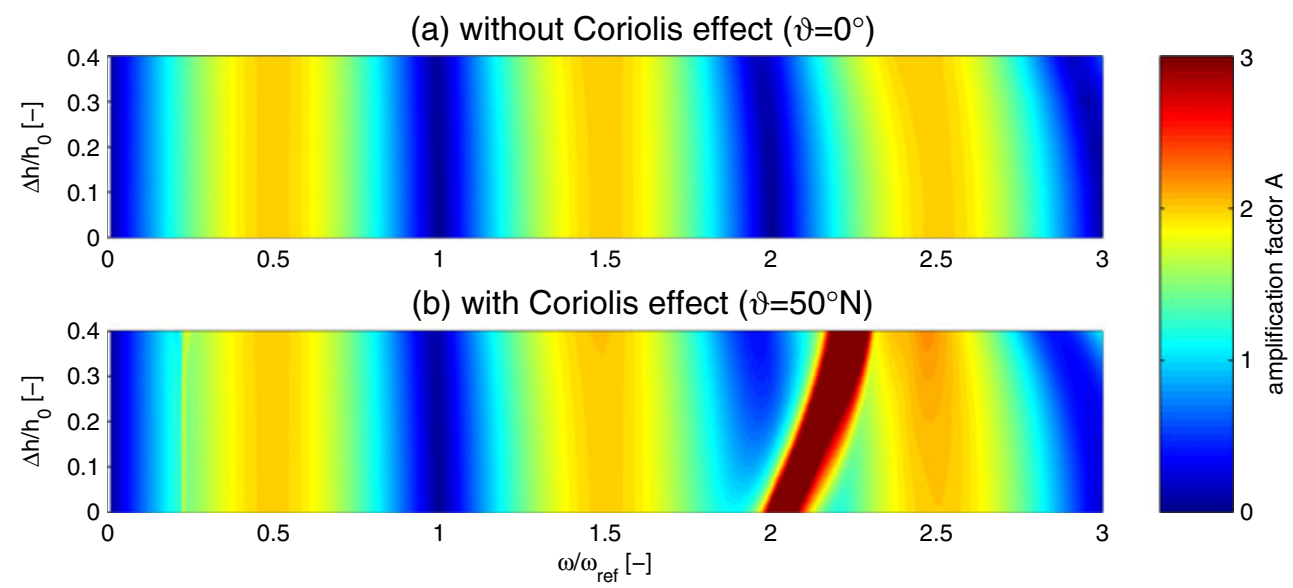


multiples of) the quarter wavelength, which are known to increase when increasing the coastal depth. Conversely, when sufficiently deep, the coastal part displays a spatially uniform elevation pattern ('pumping mode', see top panel of Fig. 5).

3. Smoothening the steps shows that increasing the slope length shifts the maximum to slightly higher frequencies.

4. The response to a linear along-basin bed profile in the inner region (along-basin wind only) shows higher response for increasing slopes (while fixing the depth in the outer region) because of the reduced depth in the coastal part.

5. The response to a parabolic cross-basin profile (alongbasin wind only) shows that varying the central depth, while keeping the average depth the same, only weakly modifies the frequency response.

The Coriolis effect causes the (strong) resonance peaks associated with cross-basin modes (which without rotation only appear in the response to cross-basin wind) to emerge also in response to along-basin wind. It also introduces peaks at the inertial frequency.

As already expressed by Eq. 1 in the introduction, a real storm event, e.g. showing a Gaussian pattern of wind stress over time, can be built up with periodic signals at different frequencies. By linearity, the response to such an event will be the superposition of the responses to these individual frequencies, weighted with the relative importance of this frequency in the event. If the resonant frequencies are strongly represented in the spectral representation of the wind event, large response will occur (Chen et al. 2015). An implication of the present study is that large-scale topographic changes, e.g. due to human intervention (dredging, land reclamation), may cause shifts in resonance peaks and, hence, changes in the basin response to wind events.

This study is a first step towards understanding the responses of natural basins to wind forcing. In particular, our modelling approach also applies to basins with a topography that is more complicated than the schematised representation used in this study. Furthermore, extending the model with respect to atmospheric forcing (e.g. representing the moving low-pressure system of a hurricane) and geometry (coastlines) is a subject of ongoing research.

Acknowledgments This work is partly funded by the Chinese Scholarship Council and partly by the research programme 'Impact of climate change and human intervention on hydrodynamics and environmental conditions in the Ems-Dollart estuary: an integrated data-modelling approach'. The latter project is financed by the Bundesministerium fur Bildung und Forschung (BMBF) and by the Netherlands Organization for Scientific Research (NWO), as part of the International Wadden Sea programme (GEORISK project). We thank Hans Burchard and two other anonymous reviewers for their comments.
Open Access This article is distributed under the terms of the Creative Commons Attribution 4.0 International License (http:// creativecommons.org/licenses/by/4.0/), which permits unrestricted use, distribution, and reproduction in any medium, provided you give appropriate credit to the original author(s) and the source, provide a link to the Creative Commons license, and indicate if changes were made.

\section{Appendix A: Ramp-up function}

The ramp-up function $\mu(x)$ of the wind describes the transition from zero wind in the outer region to a spatially uniform wind field, in a region of length $L_{\text {ramp }}$ (see Fig. 1c). It is given by

$\mu(x)= \begin{cases}0 & \text { if } x<0, \\ \frac{\sin k x}{\sin k L_{\mathrm{ramp}}} & \text { if } 0 \leq x \leq L_{\mathrm{ramp}}, \\ 1 & \text { if } x>L_{\mathrm{ramp}} .\end{cases}$

Here, $k$ is the wave number as given in Eq. 46 in Appendix D. The motivation for this particular shape of the ramp-up function, which through $k$ depends on the problem parameters, lies in the solution method. In fact, only this choice allows us to obtain analytical solutions (Appendix D) and collocation solutions (Appendix C) for the cases indicated in Section 3.3. To have a monotonically increasing ramp-up function, i.e. without any oscillations, we choose $L_{\text {ramp }}$ such that $k L_{\text {ramp }}<\pi / 2$.

\section{Appendix B: Details of the derivation}

\section{B. 1 Vertical profiles from horizontal momentum equations}

Here, we present the details of the vertical structure of the flow. First, we define rotating flow components according to $q^{ \pm}=u \pm i v$ with complex amplitudes $Q^{ \pm}$, such that $U=\left(Q^{+}+Q^{-}\right) / 2$ and $V=\left(Q^{+}-Q^{-}\right) /(2 i)$. The rotating flow solution contains three contributions, proportional to the surface gradient the wind stress and the pressure gradient, respectively:

$Q^{ \pm}(z)=Q_{\eta}^{ \pm}(z) \mathcal{L}^{ \pm} N+Q_{\mathrm{w}}^{ \pm}(z) T^{ \pm}$

with complex operators $\mathcal{L}^{ \pm}=\partial / \partial x \pm \mathrm{i} \partial / \partial y$ and rotating wind forcing amplitudes $T^{ \pm}=T^{(x)} \pm \mathrm{i} T^{(y)}$ (wind stress divided by density). The vertical structures read

$$
\begin{aligned}
& Q_{\eta}^{ \pm}(z)=\frac{g\left[\cosh \lambda^{+} z-\alpha_{\mathrm{c}}^{ \pm}\right]}{\alpha_{\mathrm{c}}^{ \pm} K \lambda^{ \pm 2}}, \\
& Q_{\mathrm{w}}^{ \pm}(z)=\frac{\alpha_{\mathrm{c}}^{ \pm} \sinh \lambda^{ \pm} z+\alpha_{\mathrm{s}}^{ \pm} \cosh \lambda^{ \pm} z}{\alpha_{\mathrm{c}}^{ \pm} K \lambda^{ \pm}} .
\end{aligned}
$$


with $\lambda^{ \pm 2}=-\mathrm{i}(\omega \mp f) / K$ and $\alpha_{c}^{ \pm}=\cosh \lambda^{ \pm} h+$ $s^{-1} K \lambda^{ \pm} \sinh \lambda^{ \pm} h$ and $\alpha_{\mathrm{s}}^{ \pm}=\sinh \lambda^{ \pm} h+s^{-1} K \lambda^{ \pm} \cosh \lambda^{ \pm} h$. The vertical integral is given by

$\left\langle Q^{ \pm}\right\rangle=\left\langle Q_{\eta}^{ \pm}\right\rangle \mathcal{L}^{ \pm} N+\left\langle Q_{\mathrm{w}}^{ \pm}\right\rangle T^{ \pm}$,

with

$\left\langle Q_{\eta}^{ \pm}\right\rangle=\frac{g\left[\sinh \lambda^{ \pm} h-\alpha_{\mathrm{c}}^{ \pm} \lambda^{ \pm} h\right]}{\alpha_{\mathrm{c}}^{ \pm} K \lambda^{ \pm 3}}$,

$\left\langle Q_{\mathrm{w}}^{ \pm}\right\rangle=\frac{\alpha_{\mathrm{c}}^{ \pm}\left[1-\cosh \lambda^{ \pm} h\right]+\alpha_{\mathrm{s}}^{ \pm} \sinh \lambda^{ \pm} h}{\alpha_{\mathrm{c}}^{ \pm} K \lambda^{ \pm 2}}$.

The two cases $\omega= \pm f$ require alternative expressions for either $Q^{+}$or $Q^{-}$. If $\omega=+f$, we must replace the $Q^{+}$expressions in Eqs. 31-33; if $\omega=-f$, we must replace the $Q^{-}$-expressions. They must be replaced with

$Q_{\eta}^{ \pm}(z)=\frac{g h^{2}}{K}\left[\frac{1}{2}\left(\frac{z}{h}\right)^{2}-\frac{1}{2}-\frac{K}{s h}\right]$,

$Q_{\mathrm{w}}^{ \pm}(z)=\left[1+\left(\frac{z}{h}\right)+\frac{K}{s h}\right]$,

and

$\left\langle Q_{\eta}^{ \pm}\right\rangle=-\frac{g h^{3}}{K}\left[\frac{1}{3}+\frac{K}{s h}\right], \quad\left\langle Q_{\mathrm{w}}^{ \pm}\right\rangle=\frac{h^{2}}{K}\left[\frac{1}{2}+\frac{K}{s h}\right]$.

\section{B. 2 Elliptical problem for $N$}

Depth-integration of the continuity Eq. 4, with the aid of boundary conditions (5) gives, in terms of the complex amplitudes of surface elevation and the rotating velocity components.

$-\mathrm{i} \omega N+\frac{\partial}{\partial x} \underbrace{\left(\frac{\left\langle Q^{+}\right\rangle+\left\langle Q^{-}\right\rangle}{2}\right)}_{\langle U\rangle}+\frac{\partial}{\partial y} \underbrace{\left(\frac{\left\langle Q^{+}\right\rangle-\left\langle Q^{-}\right\rangle}{2 \mathrm{i}}\right)}_{\langle V\rangle}=0$,

Substitution of Eq. 30 gives the elliptical equation for $N$ presented in Eq. 13 of the main text. The corresponding coefficients are given by

$C^{+}=\frac{1}{2}\left[Q_{\eta}^{+}+Q_{\eta}^{-}\right], \quad C^{-}=\frac{1}{2 \mathrm{i}}\left[Q_{\eta}^{+}-Q_{\eta}^{-}\right]$,

The boundary conditions presented in Eqs. 15-16 of the main text follow from depth-integration of the momentum Eqs. 2-3. The coefficients in there are given by

$R^{+}=\frac{1}{2}\left[Q_{\mathrm{w}}^{+} T^{+}+Q_{\mathrm{w}}^{-} T^{-}\right]$,

$R^{-}=\frac{1}{2 \mathrm{i}}\left[Q_{\mathrm{w}}^{+} T^{+}-Q_{\mathrm{w}}^{-} T^{-}\right]$.

\section{B. 3 Vertical flow velocity}

The vertical flow velocity amplitudes at any depth $z$ are given by

$$
\begin{aligned}
W(z)= & -\left\lfloor C^{\oplus}\right\rfloor\left(\frac{\partial^{2} N}{\partial x^{2}}+\frac{\partial^{2} N}{\partial y^{2}}\right) \\
& -\left\langle C^{\oplus}\right\rangle\left(\left\lfloor\frac{\partial R^{\oplus}}{\partial x}\right\rfloor+\left\lfloor\frac{\partial R^{\ominus}}{\partial y}\right\rfloor\right),
\end{aligned}
$$

where floor brackets indicate integration from bottom to $z$, i.e. $\lfloor\cdot\rfloor=\int_{-h}^{z} \cdot \mathrm{d} z$. This expression can be simplified further by using the differential Eq. 13 for $N$ to eliminate the Laplacian of $N$.

\section{Appendix C: Collocation method}

This appendix describes the collocation method that we apply for the uniform depth case, for cross-basin wind and $f=0$. The modifications necessary in the abrupt step case are mentioned at the end of the analysis. For uniform depth, we distinguish the solution in the ramp-up zone $\left(N_{1}\right)$ from that in the core of the inner region $\left(N_{2}\right)$. The problem posed in Eqs. 13-18, as derived in Appendix B, then reduces to a nonhomogeneous Helmholtz problem:

$\frac{\partial^{2} N_{0}}{\partial x^{2}}+\frac{\partial^{2} N_{0}}{\partial y^{2}}+k^{2} N_{0}=0$,

$\frac{\partial^{2} N_{1}}{\partial x^{2}}+\frac{\partial^{2} N_{1}}{\partial y^{2}}+k^{2} N_{1}=-\left[\frac{\partial\left\langle R_{1}^{+}\right\rangle}{\partial x}+\frac{\partial\left\langle R_{1}^{-}\right\rangle}{\partial y}\right]$,

$\frac{\partial^{2} N_{2}}{\partial x^{2}}+\frac{\partial^{2} N_{2}}{\partial y^{2}}+k^{2} N_{2}=0$.

Only in the ramp-up zone there is a divergence of the wind forcing, explaining the nonzero right-hand side of Eq. 44. Furthermore, $k$ is a wave number satisfying

$k^{2}=\frac{-\mathrm{i} \omega}{\left\langle C^{+} s\right\rangle}$,

with the depth-integrated coefficient $\left\langle C^{+}\right\rangle=\left\langle C_{0}^{+}\right\rangle=$ $\left\langle C_{1}^{+}\right\rangle=\left\langle C_{2}^{+}\right\rangle$(uniform depth) as specified in Eq. 40. The boundary and matching conditions remain as in Eqs. 15-18. 
Analogous to our extended FEM model in Section 3.3, the solution in the outer region is written as a superposition of outgoing wave modes, i.e.

$N_{0}(x, y)=\sum_{m=0}^{M} c_{0, m}^{\ominus} N_{m}^{\ominus}(y) \exp \left(\mathrm{i} k_{m}^{\ominus} x\right)$,

with coefficients $c_{0, m}^{\ominus}$. The cross-basin structures $N_{m}^{\ominus}(y)$ and wave numbers $k_{m}^{\ominus}$ are specified in Appendix E. The solutions in the ramp-up region and the inner region are written as a superposition of two families of wave modes plus a particular solution:

$$
\begin{gathered}
N_{j}(x, y)=N_{j, \text { part }}(x, y)+\sum_{m=0}^{M} c_{j, m}^{\oplus} N_{m}^{\oplus}(y) \exp \left(\mathrm{i} k_{m}^{\oplus} x\right) \\
+\sum_{m=0}^{M} c_{1, m}^{\ominus} N_{m}^{\ominus}(y) \exp \left(\mathrm{i} k_{m}^{\ominus} x\right),
\end{gathered}
$$

The particular solutions $N_{j \text {,part }}(x, y)$ for $j=1,2$ are introduced to homogenise the cross-basin boundary conditions. For the ramp-up zone and the core of the inner region, they are given by

$$
\begin{aligned}
& N_{1, \text { part }}(x, y)=-\left(y-\frac{B}{2}\right) \frac{\left\langle Q_{\mathrm{w}}^{+}\right\rangle+\left\langle Q_{\mathrm{w}}^{-}\right\rangle}{2\left\langle C^{+}\right\rangle} \frac{\sin k x}{\sin k L_{\mathrm{ramp}}}, \\
& N_{2, \text { part }}(x, y)=-\frac{1}{k}\left(\sin k y-\frac{1-\cos k B}{\sin k B} \cos k y\right) \frac{\left\langle Q_{w}^{+}\right\rangle}{\left\langle C^{+}\right\rangle} .
\end{aligned}
$$

The solution in Eqs. 47-48 is thus contained in the five families of in total $5(M+1)$ coefficients $c_{0, m}^{\ominus}, c_{1, m}^{\oplus}, c_{1, m}^{\oplus}$, $c_{2, m}^{\oplus}$, and $c_{2, m}^{\oplus}$ (for $m=0,1, \cdots, M$ ). Their values follow from applying a collocation technique similar to that applied by Roos and Schuttelaars (2011). To this end, we require the matching of surface elevation and normal transport to be satisfied at two sets of $M+1$ collocation points at $x=0$ and $x=L_{\text {ramp }}$, and the closed boundary condition at another set of collocation points $x=L$.

The analysis for the abrupt step case requires dividing the inner region into three parts: the ramp-up zone denoted with subscript $j=1$, an offshore part of depth $h_{0}(j=2)$ and a coastal part of depth $h_{\mathrm{c}}(j=3)$. The above analysis can then be repeated, distinguishing a different wave number in the coastal part and imposing similar matching conditions also at $x=x_{\text {step. }}$. For brevity, the analysis is not presented here.

\section{Appendix D: 2DV-solution}

This appendix contains the analytical 2DV solution without rotation $(f=0)$, with along-basin wind $(\theta=0)$, for the uniform depth. The modifications necessary in the abrupt step case are mentioned at the end of the analysis. This solution is termed ' $2 \mathrm{DV}$ ' because there is neither flow in the crossbasin direction nor dependency on the cross-basin coordinate $y$. Hence, the flow amplitudes depend on $x$ and $z$ only, and the elevation amplitude $N(x)$ depends on the alongbasin coordinate only. The problem posed in Eqs. 43-45, as presented in Appendix $\mathrm{C}$ for the uniform depth case, now reduces to a one-dimensional Helmholtz problem. We thus write

$$
\begin{aligned}
& \frac{\partial^{2} N_{0}}{\partial x^{2}}+k^{2} N_{0}=0, \\
& \frac{\partial^{2} N_{1}}{\partial x^{2}}+k^{2} N_{1}=-\frac{\partial\left\langle R_{1}^{\oplus}\right\rangle}{\partial x}, \\
& \frac{\partial^{2} N_{2}}{\partial x^{2}}+k^{2} N_{2}=0 .
\end{aligned}
$$

Only in the ramp-up region there is a divergence of the wind forcing, explaining the nonzero right-hand side of Eq. 52. The wave number $k$ is still as given by Eq. 46 in Appendix $\mathrm{C}$, and the boundary and matching conditions in Eqs. $15-18$ become

$\left\langle C_{2}^{\oplus}\right\rangle \frac{\mathrm{d} N_{2}}{\mathrm{~d} x}=-\left\langle R_{2}^{\oplus}\right\rangle$,

at $x=L$ and

$N_{j}=N_{j+1}, \quad \frac{\mathrm{d} N_{j}}{\mathrm{~d} x}=\frac{\mathrm{d} N_{j+1}}{\mathrm{~d} x}$,

to be satisfied at $x=0$ for $j=0$ and at $x=L_{\text {ramp }}$ for $j=1$. The solution is given by

$N_{0}=a \exp (-\mathrm{i} k x)$

$N_{1}=a \exp (-\mathrm{i} k x)-c \frac{k x \sin k x}{\sin \varphi_{\text {ramp }}}$

$N_{2}=b \cos (k[x-L])-2 c \sin (k[x-L])$,

with coefficients $a, b$ and $c$ given by

$$
\begin{aligned}
a= & \frac{\left\langle Q_{w}^{+}\right\rangle \hat{T}}{2 k\left\langle C_{1}^{\oplus}\right\rangle} \\
& \times\left[\frac{\varphi_{\text {ramp }}\left(\tan \Delta \varphi-\cot \varphi_{\text {ramp }}\right)-1}{(\tan \Delta \varphi-\mathrm{i}) \exp \left(\mathrm{i} \varphi_{\text {ramp }}\right)}+2 \mathrm{i} \exp (-\mathrm{i} \varphi)\right], \\
b= & \frac{\left\langle Q_{w}^{+}\right) \hat{T}}{2 k\left\langle C_{1}^{\oplus}\right\rangle} \\
& \times\left[-\mathrm{i} \exp (-\mathrm{i} \Delta \varphi)\left(1+\varphi_{\mathrm{ramp}} \cot \varphi_{\mathrm{ramp}}-\mathrm{i} \varphi_{\mathrm{ramp}}\right)+2 \mathrm{i}\right], \\
c= & \frac{\left\langle Q_{w}^{+}\right) \hat{T}}{2 k\left\langle C_{1}^{\oplus}\right\rangle},
\end{aligned}
$$

with $\varphi=k L, \varphi_{\text {ramp }}=k L_{\text {ramp }}$, and $\Delta \varphi=k\left(L-L_{\text {ramp }}\right)$.

The analysis for the abrupt step case requires dividing the inner region into three parts: the ramp-up zone denoted with subscript $j=1$, an offshore part of depth $h_{0}(j=2)$ and a 
coastal part of depth $h_{\mathrm{c}}(j=3)$. The above analysis can then be repeated, distinguishing a different wave number in the coastal part and imposing similar matching conditions also at $x=x_{\text {step }}$. For brevity, the analysis is not presented here.

\section{Appendix E: Wave modes}

Our solution technique makes use of families of so-called Kelvin and Poincare modes, which are eigenmodes in an infinite channel of uniform depth and width. We distinguish two families: those propagating (or exponentially decaying) in the positive $x$-direction (termed 'positive' modes, indicated with superscript $\oplus$ ) and those propagating (or exponentially decaying) in the negative $x$-direction (termed 'negative' modes, indicated with superscript $\ominus$ ).

More precisely, the solution in the outer region, as given by Eq. 20, is written as a truncated superposition of 'negative' Kelvin and Poincaré modes. Furthermore, the collocation technique described in Appendix $\mathrm{C}$ uses eigenmodes also in the ramp-up and inner region. This involves both positive and negative modes, but only in the $f=0$ limit.

For arbitrary $f$, the Kelvin mode, propagating in the positive $x$-direction, is given by

$$
\begin{aligned}
N_{0}(y)^{\oplus} & =\exp \left(\frac{-y}{R_{\text {def }}}\right) \exp \left(\mathrm{i} k_{0}^{\oplus} x\right), \\
R_{\text {def }} & =\sqrt{\frac{\left\langle\left(C_{j}^{+}\right)^{2}\right\rangle+\left\langle\left(C_{j}^{-}\right)^{2}\right\rangle}{\left\langle\left(C_{j}^{+}\right)^{2}\right\rangle k^{2}} .}
\end{aligned}
$$

with wave number

$k_{0}^{\oplus}=\sqrt{\frac{\left\langle\left(C_{j}^{-}\right)^{2}\right\rangle k^{2}}{\left\langle\left(C_{j}^{+}\right)^{2}\right\rangle+\left\langle\left(C_{j}^{-}\right)^{2}\right\rangle}}$.

The Kelvin mode propagating in the negative $x$-direction is obtained by a symmetry argument, which also implies $k_{0}^{\ominus}=-k_{0}^{\oplus}$. In the $f=0$ limit, for which $\left\langle C_{j}^{-}\right\rangle=0$, the Kelvin mode effectively becomes a shallow water wave with a uniform cross-basin structure.

The Poincaré modes, propagating or exponentially decaying in the positive $x$-direction, are given by

$N_{m}^{\oplus}(x, y)=\left[\cos \left(\frac{m \pi y}{B}\right)-\frac{\mathrm{i} k\left\langle C_{j}^{\oplus}\right\rangle}{m \pi\left|C_{j}^{\ominus}\right\rangle} \sin \left(\frac{m \pi y}{B}\right)\right] \exp \left(\mathrm{i} k_{m}^{\oplus} x\right)$,

for $m=1,2, \cdots$ and with the wave number $k_{m}^{\oplus}$ satisfying

$k_{m}^{\oplus 2}=k^{2}-\left(\frac{m \pi}{B}\right)^{2}$.

The Poincaré modes propagating in the negative $x$-direction are obtained by a symmetry argument, which also implies $k_{m}^{\ominus}=-k_{m}^{\oplus}$. In the limit $f=0$, the Poincaré modes obtain an elevation structure proportonal to $\cos (m \pi y / B)$.

For brevity, the along-basin flow components of the above modes are not presented here.

\section{References}

Abraham G (1960) Hurricane storm surge considered as a resonance phenomenon. Coast Eng Proc 1(7):31. https://icce-ojs-tamu.tdl. org/icce/index.php/icce/article/view/2190

Breaker LC, heng Tseng Y, Wang X (2010) On the natural oscillations of monterey bay: observations, modeling, and origins. Prog Oceanogr 86(34):380-395. doi:10.1016/j.pocean.2010.06.001

Büsching F (2003) Storm wave resonance controlled by hollow block structure. In: Proceedings of 6th international conference on coastal and port engineering in developing countries COPEDEC VI, Colombo, pp 1-20,90

Chernetsky AS, Schuttelaars HM, Talke AS (2010) The effect of tidal asymmetry and temporal settling lag on sediment trapping in tidal estuaries. Ocean Dyn 60(5):1219-1241. doi:10.1007/s10236-010-0329-8

Chen Q, Wang L, Tawes R (2008) Hydrodynamic response of northeastern Gulf of Mexico to hurricanes. Estuar Coasts 31(6):10981116. doi:10.1007/s12237-008-9089-9

Chen WL, Roos PC, Schuttelaars HM, Hulscher SJMH (2015) Resonance properties of a closed rotating rectangular basin subject to space- and time-dependent wind forcing. Ocean Dyn 65(3):325339. doi:10.1007/s10236-015-0813-2

Craig PD (1989) Constant-eddy-viscosity models of vertical structure forced by periodic winds. Cont Shelf Res 9(4):343-358. doi:10.1016/0278-4343(89)90038-1

Csanady GT (1968) Wind-induced barotropic motions in long lakes. J Phis Oceanogr 3:429-438. doi:10.1175/1520-0485(1973)003 $<0429$ :WIBMIL $>2.0$.CO;2

Gockenbach MS (2006) Understanding and implementing the Finite Element Method Society of industrial and applied mathematics. SIAM, Philadelphia

Hanley M, Hoggart S, Simmonds D, Bichot A, Colangelo M, Bozzeda F, Heurtefeux H, Ondiviela B, Ostrowski R, Recio M, Trude R, Zawadzka-Kahlau E, Thompson R (2013) Shifting sands? Coastal protection by sand banks, beaches and dunes. Coast Eng 87:136146. doi:10.1016/j.coastaleng. 10.020

Hulscher SJMH (1996) Tidal-induced large-scale regular bed form patterns in a three-dimensional shallow water model. J Geophys Res 101(c9):20727-20744. doi:10.1029/96JC01662

Irish JL, Resio DT, Ratcliff JJ (2008) The influence of storm size on hurricane surge. J Phys Oceanogr 38(9):2003-2013. doi:10.1175/2008JPO3727.1

Kumar M, Schuttelaars HM, Roos PC, Möller M (2015) Threedimensional semi-idealized model for tidal motion in tidal estuaries. An application to the Ems estuary. Ocean Dyn 66(1):99-118. doi:10.1007/s10236-015-0903-1

Libicki C, Bedford KW (1990) Sudden, extreme lake erie storm surges and the interaction of wind stress, resonance, and geometry. J Great Lakes Res 16(3):380-395. doi:10.1016/S0380-1330(90)71432-3

Moon IJ, Oh IS, Murty T, Youn YH (2003) Causes of the unusual coastal flooding generated by typhoon Winnie on the west coast of Korea. Nat Hazards 29(3):485-500. doi:10.1023/A:1024798718572

Mathieu PP, Deleersnijder E, Cushman-Roisin B, Beckers JM, Bolding $\mathrm{K}$ (2002) The role of topography in small well-mixed bays, 
with application to the lagoon of Mururoa. Cont Shelf Res 22(9):1379-1395. doi:10.1016/S0278-4343(02)00002-X

Mass LRM, Van Haren JJM (1987) Observations on the vertical structure of tidal and inertial currents in the Central North Sea. J Mar Res 45(2):293-318. doi:10.1357/002224087788401106

Ponte AL (2010) Periodic wind-driven circulation in an elongated and rotating basin. J Phys Oceanogr 40(9):2043-2058. doi:10.1175/2010JPO4235.1

Ponte AL, de Velasco GG, Valle-Levinson A, Winters KB, Winant CD (2012) Wind-driven subinertial circulation inside a semienclosed bay in the Gulf of California. J Phys Oceanogr 42(6):940-955. doi:10.1175/JPO-D-11-0103.1

Proudman J (1929) The effects on the sea of changes in atmospheric pressure. Mon Not R Astr Soc Geophys Suppl 2(4):197

Rabinovich AB (2009) Seiches and harbor oscillations. In: Handbook of coastal and ocean engineering, SpringerBriefs in Environmental Science, World Scientific, chap 9, pp 193-236
Rao DB (1966) Free gravitational oscillations in rotating rectangular basins. J Fluid Mech 25(3):523-555. doi:10.1017/S0022112066 000235

Roos PC, Schuttelaars HM (2011) Influence of topography on tide propagation and amplification in semi-enclosed basins. Ocean Dyn 61(1):21-38. doi:10.1007/s10236-010-0340-0

Sobey RJ (2005) (2006) Normal mode decomposition for identification of storm tide and tsunami hazard. Coast Eng 53(23):289-301. doi:10.1016/j.coastaleng. 10.016

Weaver R, Slinn D (2010) Influence of bathymetric fluctuations on coastal storm surge. Coast Eng 57(1):62-70. doi:10.1016/j.coastaleng.2009.09.012

Wilson BW (1972) Seiches. Advances Hydrosci. 8:1-94

Winant CD (2004) Three-dimensional wind-driven flow in an elongated, rotating basin. J Phys Oceanogr 34(2):462476. doi:10.1175/1520-0485(2004)034<0462:TWFIAE $>2.0$. 